\title{
Choices and Choosing in Cancer Genetics
}

\author{
Dirk Stemerding and Annemiek Nelis ${ }^{1}$
}

\section{Introduction}

Clinical genetics has established itself as a medical practice in which patients are addressed primarily as individuals who have to make informed choices (Bosk 1992; Steendam 1996). Historically, centres for clinical genetics in the Netherlands have a privileged position in offering genetic counselling and genetic testing, and function as "gatekeepers" for those seeking genetic consultation and diagnosis (Nelis 2000). In these centres, genetic diagnosis is embedded in a practice of counselling in which facts deemed relevant are separated out as "information" that is handed out to clients along with a few courses of action formulated as possible alternatives and between which clients are requested to choose. The establishment of clinical genetics thus created a particular space for individual decision making in which, from the 1990 onwards, new options for genetic testing on the basis of DNA-diagnosis have been introduced and evaluated primarily as bringing along new opportunities of choice (Bourret et al. 1998).

In today's health care, this particular configuration - that of the individual patient called upon "to decide" - is held in high esteem. A good doctor leaves patients room for choice. But how can we understand the issue of individual choice when we simultaneously consider the rapidly developing practices of medical genetics from a perspective of co-production of technology and society? From this perspective, we are interested in the question of how technological objects and individual subjects - nonhuman and human agents - mutually shape one another in an emerging web of socio-technical relations (Berg and Mol 2001). Looking at recent developments in medical genetics in this way, we can make two observations. We see the emergence of increasingly complex networks, involving a growing interdependency between many different actors: technologies of screening, registries, medical specialists, patients, clinical genetics centres, and individuals at risk. At the same time, we 
see how in this network a particular model of human agency is endorsed, according to which it is the individual patient or person at risk who has to decide.

How should one weigh these observations? Should we interpret them as revealing a tension or even contrast between "structure" - embodied in networks and interdependencies - and "action" - embodied in individual freedom of choice? Or should we understand the model of individual decision making as a particular outcome of a process of co-production of technology and society? Framed in this way, our observations invite us to go beyond "an oscillation in social analysis between action as determining and action as determined" (Gomart and Hennion 1999). That is, we are invited to shift the analysis to the collective production of human agency, to the creation of particular subject positions as an effect of emergent socio-technical networks.

In this chapter, we attempt to take up this invitation in an analysis of how subjects and responsibilities are constituted in new emerging practices of screening in the field of cancer genetics. We focus our study on the way in which in the Netherlands a screening practice has emerged for familial adenomatous polyposis (FAP), a specific hereditary predisposition for colon cancer. First, we shall show how in the 1980 os a regular screening practice was created which involved both patients and "individuals at risk". Then we describe how this screening practice was extended with new elements, with the development and introduction of DNA-diagnostic tests, involving new choices and responsibilities. Thus, we shall cover the way in which responsibilities were distributed and human agency was shaped in these various contexts. In conclusion, we shall discuss our findings in the context of current debates about patient autonomy and freedom of choice in today's health care and the emerging new genetics.

\section{FAP in the early 1980s: the emergence of a screening practice}

In 1981 a collection of three articles and a commentary appeared in an issue of the Dutch Journal of Medicine, which focussed on the clinical experiences involving a rare, dominantly hereditary disease, called polyposis coli or familial adenomatous polyposis (FAP). In each of the three articles, an elaborate description was given of the history of the disease in a particular family. One of the articles opened with the story of a 27-year-old woman who consulted the clinic because a 34-year-old cousin of hers had been recently identified as an FAP patient after the diagnosis of colon cancer (De Ruiter and Den Hartog Jager 1981). The mother of the woman had died of colon cancer when she was 
43, a few years after the colon had been partially removed because of a malignant polyposis. The authors of the article observed with regret that clinical examinations of relatives had not been undertaken at the time, although it was known that a grandmother and a great-grandfather had also died from "cancer of the colon". The woman who was consulting the clinic had no symptoms, but inspection of the colon revealed many polyps resulting in a subsequent diagnosis of FAP and in the decision to completely remove the colon. The article then continued with the case of a younger sister of the woman, who similarly displayed no symptoms, but who also had her colon removed after investigation had indicated polyposis. Other brothers and sisters examined only revealed a few polyps in the colon. In these cases, as the authors point out, the examination had to be repeated every year. In conclusion, the article reports the results of examinations that had been carried out on 39 people, spanning two generations of the family. Polyposis was found in 12 cases, and colon cancer had already developed in 4 of them. Four people refused the invitation to undergo examination.

The collective publication of the three articles and the accounts given by the authors of the diagnosis, treatment and screening in families where FAP is found may be seen as an event which marks the emergence of a specific clinical practice, i.e., a practice in which the professional responsibility of the medical specialist - an internist or gastro-enterologist - cannot be restricted to the individual patient, but should also extend to the health and survival of the patient's relatives (see figure 1 below). When a patient finally consults the clinic with specific symptoms and the diagnosis of FAP is made, in most cases a fatal colon cancer will have already appeared. Thus, as the authors of the articles point out, it is of vital importance to trace the families in which FAP is found and to screen the members of these families every two or three years,

Figure 1: Emergent cancer genetic practice in the field of polyposis (late 1970s)

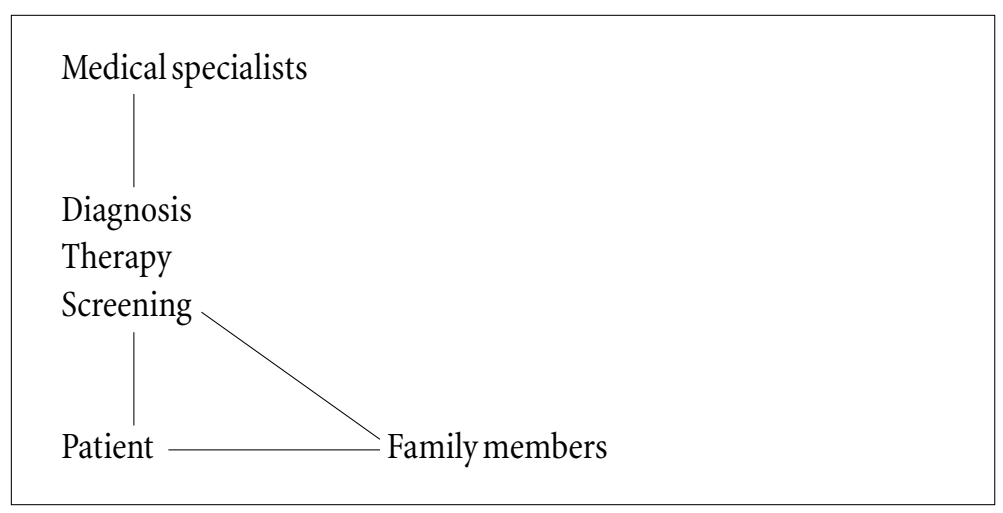


beginning from about the age of ten. As soon as more than one hundred polyps are found in the colon, the diagnosis of FAP should be made, and it is only by complete removing the colon that the development of cancer can be prevented. Even then, regular screening remains necessary. If, on the other hand, family members are still free of symptoms between the ages of forty and fifty, the appearance of polyposis can be reasonably counted out, and screening may be terminated.

The conclusions and recommendations of the authors were clearly intended to promote a practice that, at the beginning of the 1980s, was still in its infancy. At that time, it was already considered against due practice to refrain from an extensive family anamnesis when the diagnosis of FAP had been made. However, in the preceding period, this kind of family anamnesis was by no means the rule, which is explained, in one of the articles, by a general lack of knowledge of the serious consequences of the disease. Even when an extensive mapping of the family history of the patient followed the diagnosis of FAP, it was often considered to be an impossible task for the individual specialist to actively approach all the family members involved. And, as far as family members were actually approached, the specialist could not always be sure that they would return regularly for periodic screening. Thus, in a commentary on the three articles mentioned above, published in the same issue of the Dutch Journal of Medicine, it was observed that:

Detective-like genealogical investigations, the psychological burden experienced by people who feel completely healthy and yet face the prospect of invasive examinations of the colon, and the not always interesting task to screen a fairly large number of people who have no symptoms, require a great and unremitting enthusiasm and dedication of those who undertake to follow a family with a history of polyposis. (Van Slooten 1981)

Hence, the author of the commentary argued for the establishment of a centralised national registry, which could send out a reminder to medical specialists each time a person at risk had to be called in for screening. The results of the screening were to be returned to the registry. If no results followed, the organisation could take further action in order to safeguard the care for those at risk and to obtain certainty about their conditions. With his plea, the author actually repeated a message that he had already voiced in the same journal no less than 25 years earlier. This time, however, the argument would find an audience. 


\section{Late 1980s: The establishment of a national registry}

At the end of the 1980s, a patient consulting a clinic with symptoms of FAP would encounter a practice that was indeed different from what we have seen earlier. The medical specialist in attendance now not only had to inform the patient about the hereditary nature of the disease and the importance of screening family members, but also could refer the patient to the national Foundation for the Detection of Hereditary Tumours. In 1983, this foundation was established by a number of specialists involved in the treatment of patients and their families suffering from hereditary tumours (one of the founders was the author of the commentary quoted above). In 1985, the Foundation started a national registry of families with a history of FAP, thus aiming to promote screening in high-risk families, to guarantee the continuity of screening, to collect data for scientific purposes, and to offer advice about the diagnosis, treatment, methods of screening, and genetic services for counselling (Vasen et al.1988).

The result of this development was a more extended practice of diagnosis, treatment and screening of FA $\mathrm{P}$, in which every patient is reported by medical specialists to the Foundation for the Detection of Hereditary Tumours (see figure 2 below). A social worker at the Foundation then approaches the patient and with his or her help draws up a family tree which makes it possible to trace the history of the disease and to identify members of the family who are at risk. The patient is asked to inform relatives at risk and to urge them to have

Figure 2: Establishment of a national registry in the field of polyposis (late 1980s)

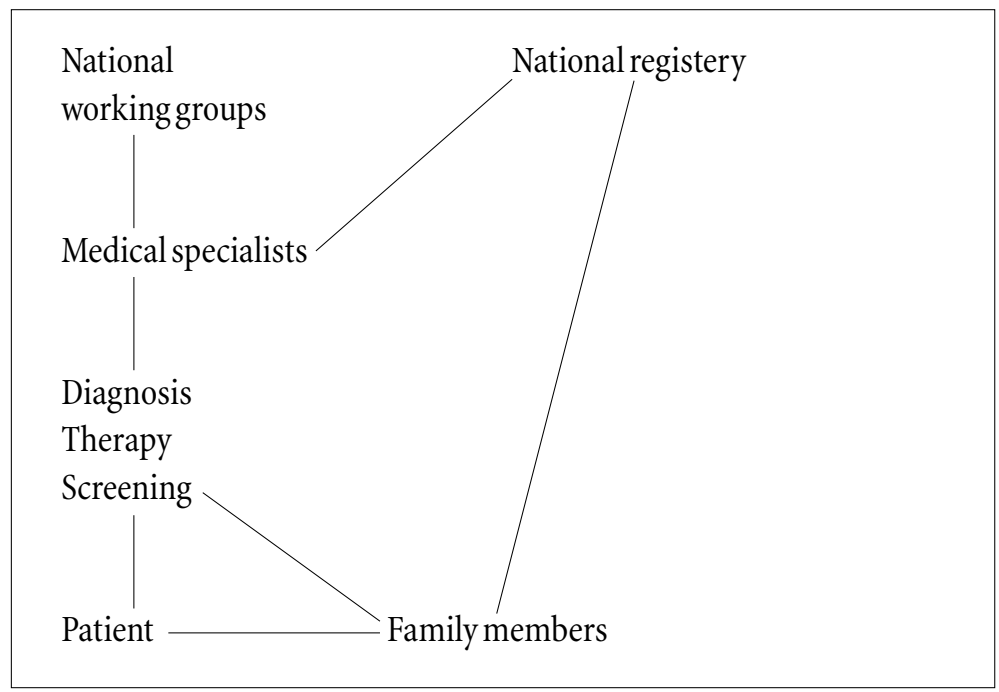


themselves screened. If they agree, family members are approached by the Foundation with a request for registration. In this way, nearly all FA P families in the Netherlands have been registered, amounting to a few hundred (Annual report 1994). Personal and medical information is collected from those who have registered, and through a system of reminders, specialists are notified when individuals should be called in for screening. If no screening results are reported, and upon inquiry it appears that someone did not turn up for screening, the registry will send out a request to the family doctor to take action and to remind this person that screening is of vital importance.

While the care of individuals with a risk of FAP initially strongly depended on the enthusiasm and efforts of individual specialists and on the awareness of those at risk, it is now the Foundation for the Detection of Hereditary Tumours which has assumed the responsibility for the organisation and continuity of screening and which "will put all efforts into encouraging (registered) individuals to comply (with regular screening)" (Annual report 1994, 5). Indeed, as those working at the Foundation point out, in order to motivate family members to participate in a screening program, good information and a personal approach including home visits are necessary (interview data, see footnote 8). Moreover, through the establishment of a national registry, information is collected which not only facilitates the organisation of a screening program, but which also creates possibilities for a systematic follow-up and an evaluation of its results. For that purpose, two national working groups on FAP, involving various forms of expertise, collaborate with the Foundation in the organisation of studies and the establishment of guidelines (Vasen et al. 1988). Thus, through the efforts of the Foundation, local practices of early detection and prevention have become part of a larger network in which these practices are organised and regulated on a national scale.

\section{Early 1990s: The advent of DNA-diagnosis}

In the early 1990s, a patient who consulted the clinic with FAP symptoms would again encounter a practice that had been extended with new elements - new technologies, rules and organisations (Vasen and Müller 1991). In 1991, molecular biologists succeeded in relating the occurrence of FAP to mutations in a particular gene, the so-called APC (adenomatous polyposis coli) gene. This finding made available the possibility of presymptomatic DNA-diagnosis whereby members of an FAP family could be informed about their individual riskstatus on the basis of mutation analysis, that is, whether they will get the disease or not. However, in the Netherlands, DNA-diagnosis is made 
available only through a network of regional clinical genetics centres. Thus, with the advent of the DNA-diagnosis of FAP, molecular biology laboratories and clinical genetic centres became part of the network in which the practice of diagnosis, treatment, and screening of FAP took shape (see figure 3 below).

A new patient will now not only be reported by a medical specialist to the Foundation for the Detection of Hereditary Tumours, but will also be referred to a clinical genetics centre for mutation analysis. Again, a clinical genetic centre counsellor will draw up a family pedigree in order to identify relatives who may be at risk of developing the disease. Family members of the family then have the opportunity (after being been informed by the patient) to be referred to a clinical genetics centre, which may offer them presymptomatic DNA-diagnosis as soon as a mutation is found. Those who accept the offer and are diagnosed as carriers know for certain that they will develop FA P and that regular screening is the only way to escape from an early and deadly cancer. Of those diagnosed as carriers, most if not all will have themselves registered in the national registry at the Foundation for the Detection of Hereditary Tumours. Those who are diagnosed as non-carriers, however, are excluded from risk and thus may abstain from participating in a burdensome and protracted screening program. In other words, DNA-diagnosis made it possible to divide a known population at risk of contracting FA P into a carrier group which can be followed with traditional clinical screening methods, and a non-carrier group which may be excluded from risk and relieved of participation in a screening program. For those who appear to be carriers, DNA-di-

Figure 3: Introduction of DNA-diagnosis in the field of polyposis (1990s)

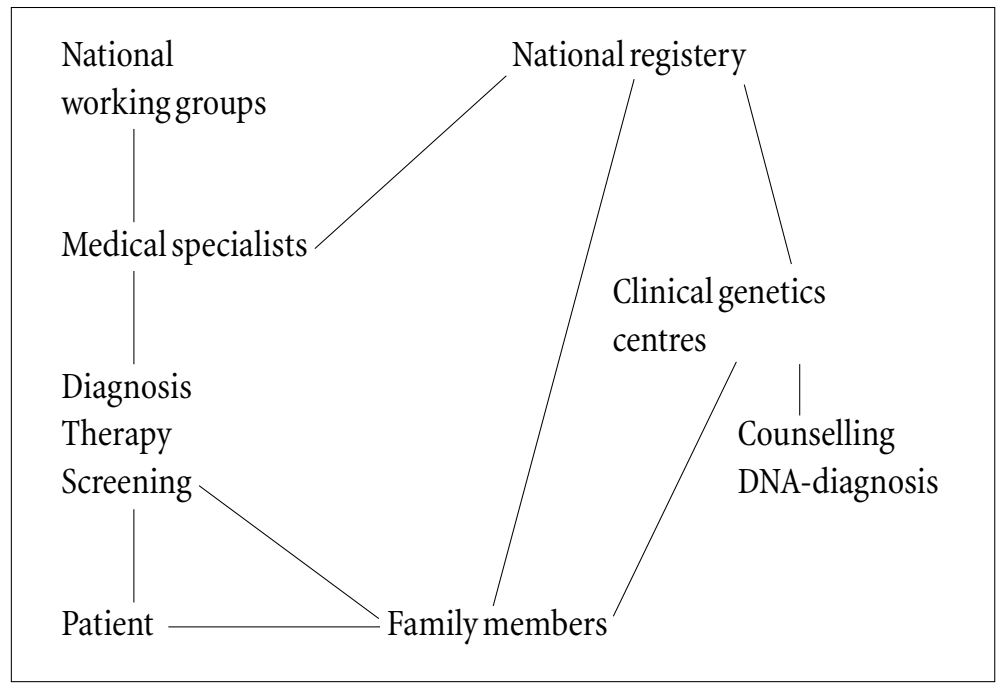


agnosis may have additional value in decisions about prophylactic interventions, and is available in the form of prenatal diagnosis. ${ }^{2}$ Thus, options for DNA-diagnosis were readily incorporated in clinical practice as a diagnostic tool which contributes to more efficient and improved forms of preventive care.

\section{A network of co-existent regimes}

In the previous sections we described the emergence of a national screening program in the field of cancer genetics as a process of co-evolution, showing changing configurations of artifacts, practices, professions, users and institutions in which a particular case of "new genetics" has been gradually taking shape. We have described these changing configurations as an extending network, that involved new actors - family members potentially at risk, social workers, a national registry, clinical geneticists - and established new alignments between these actors. In this network, the provision of information to family members about the hereditary nature of the disease, the establishment of family trees, the collection of medical data, the offer of DNA-diagnosis and clinical screening became standard elements of the responsibilities of the medical specialist, social worker, or clinical geneticist. The data collected through this network and these efforts, by the Foundation for the Detection of Hereditary Tumours, facilitated not only the organisation of a national screening program, but also the systematic monitoring of its effects and the development of guidelines to be observed in practices of diagnosis and screening. Thus, the extending FA P-network embodied a long-envisaged task to improve the management of polyposis as a hereditary disease.

Our previous account of the emergence of an FAP-network not only describes the mutual shaping or co-evolution in which FAP was transformed from a "fatal disease of the colon" into a "hereditary disorder with preventable consequences". It also shows how this process of co-evolution crystallised into a specific pattern of roles and responsibilities that the actors involved found difficult to deny. In the 1980s, actions and interactions in the evolving FAP-network both shaped and were shaped by what we might call a new regime of prevention. ${ }^{3}$ In the early 1980 s, as the publications in the Dutch Journal of Medicine show, medical specialists were already expected to inform FAP patients about the hereditary nature of the disease and the consequent implications for family members. The responsibility of the physician no longer stopped with the treatment of a patient, but also extended to the patient's family. Relatives had to be informed about potential risks and if necessary, ac- 
cording to the ruling standards of the time, considered for regular screening. With the establishment of a national registry, the responsibility of individual specialists to offer information and care to the family of patients became institutionalised on a more collective level in the working practices, database and protocols of the Foundation for the Detection of Hereditary Tumours. In other words, through the efforts of the Foundation, practices of early detection and prevention no longer depended primarily on local initiatives, but had become part of a larger cancer genetic regime of prevention.

When, in the early 1990s, researchers in Leiden found genetic markers on both sides of the so-called A P C gene, it became possible to identify gene carriers in families at risk through linkage studies (no direct mutation analysis was possible yet). However, the provision of a DNA-test did not come within the province of the gastro-enterologist or the Foundation for the Detection of Hereditary Tumours. It was the Leiden Centre for Clinical Genetics that organised and facilitated the introduction of DNA-diagnosis in FAP families. As we have already noted in the introduction, centres for clinical genetics in the Netherlands have a privileged position in offering genetic counselling and genetic testing, and in these centres, genetic diagnosis is offered through a practice of counselling in which the autonomous decision making of patients and individuals at risk is the guiding principle. When providing information, counsellors consider it as their task to be neutral and non-directive. ${ }^{4}$ The responsibility for decisions and actions to be taken is delegated primarily to the individual asking for information and advice. In the practice of genetic counselling, the principle of informed decision making is also upheld by the relatively long time available for each consult, the obligatory time-frames between consults when clients have to make important decisions, and the extensive documentation of consultations that counselors provide to their clients.

In other words, when DNA-diagnosis became available for those at risk of contracting FAP, it was embedded in a practice of clinical genetics that already constituted a regime of its own. In this regime, self-determination was the guiding principle that defined the roles and responsibilities of the actors involved (Nelis 1998 and 1999). In this context, it is interesting to see how medical specialists, in the early 1990s, considered the prospects of DNA-diagnosis in the field of cancer genetics. They referred, first of all, to the promise of improved forms of preventive care, but also pointed out that genetic counselling would deserve particular attention because those at risk of contracting (rare) hereditary tumours would have to face more complex choices (Vasen and Müller 1991). This comment, no doubt, strongly reflected the history and position of clinical genetics as a practice in which DNA-diagnosis was made available to patients and individuals at risk primarily as an opportunity of 
(informed) choice. In other words, in our account of the emerging FAP-network, the introduction of options for DNA-diagnosis not only involved the extension of this network with a few new elements, but also created a situation of co-existence in this network of two different regimes. Indeed, from the 1990 on onwards, FAP patients and individuals at risk found themselves being addressed in different ways: as subjects who need preventive care and as subjects who have to deal with (new) opportunities of choice.

\section{Ethical norms: External standards or constitutive elements?}

From the perspective of more traditional medical ethics, one might argue that our description of a regime of prevention reveals a paternalistic approach towards the choices of individuals at risk, while in a regime of self-determination, professionals rightfully comply to the principles of autonomy and informed consent as defined both by ethical and legal standards. ${ }^{5}$ The medical ethics perspective differs in a number of ways from the perspective that we try to develop in this chapter. Most importantly, within the tradition of medical ethics, norms such as patient's rights to free choice and autonomous decision making are defined as external to medical practices, that is, as universal standards which are supposed to guide and direct the daily activities of medical professionals. In contrast to this view, we want to argue that medical standards, rather than being external and given, are constitutive elements being co-produced within medical practices.

As empirically oriented philosophers and sтs-scholars have argued, patient autonomy, conceived as a universal normative ideal or ethical standard, may be difficult to localise in current everyday practices of medical care. ${ }^{6}$ For example, in an empirical study of decision making practices in a hospital setting, Schermer concludes that decision making in daily care-settings is an ongoing and diffuse process. Decisions are rarely made at one time, in one location or by one person, let alone by "the" individual patient. On the contrary, "there were many moments, with many smaller and bigger decisions clustering together" that jointly produced a particular trajectory or action (Schermer 2001, 9). What we see then is a variety of incidental decisions which are not so much geared towards the question "what does this patient want?" but to the question "what is best for this patient?". According to Schemer, that does not necessarily imply that there is a lack of respect for the autonomy of patients in hospital practices. Rather, one could say there are different meanings of autonomy that shape the relationship between the patient and the medical professionals. ${ }^{7}$ Autonomy has "different faces". 
Schermer's argument may be read as a critical evaluation of everyday medical practices which takes the normative ideal of patient autonomy as a starting-point. However, we may also understand her observations from a different point of view in which norms are not taken as ideals coming prior to the reality of everyday practices, but as being co-produced in the activities, rules, routines and procedures in which such practices take shape. From this perspective, Schermer's argument also applies to our description of different regimes in the FAP-network, showing how particular subject positions - be it the autonomous patient or the patient needing good care-emerge in various medical practices.

\title{
Well-being versus autonomy
}

Schermer's description of the practices and routines of hospital care obviously has much in common with our previous account of the ways in which patients and individuals at risk move through a FA P-network constituting what we have called a regime of prevention. In accordance with Schermer's observation that "it seemed as if considerations concerning a patient's well-being were far more important than considerations concerning patient autonomy" (82), we find in this regime of prevention a strong focus on the well-being of individuals and families at risk. Indeed, the primary aim of the Foundation for the Detection of Hereditary Tumours is to guarantee optimal care, and so it does everything in its power to encourage individuals to co-operate. In this context, the notion of choice only appears as a valuable opportunity or as a boundary that one should respect. As one of the social workers of the Foundation explains:

\begin{abstract}
Because of privacy regulations we are not allowed to approach members of the family without their personal consent. Thus, patients are invited by the Foundation to inform relatives at risk. Personal contact with patients at home makes it easier to persuade them that it is necessary to inform other members of the family and also to convince them of the benefit of screening. Sometimes, when patients are reluctant, the family doctor is called in. If members of the family don't want to be informed, then there is nothing more to be done.
\end{abstract}

Although professionals within the regime of prevention thus occasionally refer to the notion of individual choice as a valuable and indispensable opportunity, the organisation of screening and medical decision making in the FAP-network is governed by judgements, routines and standards in which the 
medical well-being of patients is clearly assigned priority over other considerations.

This is not to say, however, that individual autonomy only exists as a normative ideal to be confronted with the reality of day-to-day medical practices. Individual autonomy as a standard may also become part of everyday reality, as in the regime of self-determination constituted by the history and practice of clinical genetics. In this practice too, the roles and responsibilities that are ascribed to patients and individuals at risk are shaped by routines and standards indicating the best course of action in the process of medical decision making. Thus, when an individual at risk of contracting polyposis consults a genetic centre for DNA-diagnosis, the counsellor will meet this person on the basis of a specific protocol. In the words of a counsellor:

According to the protocol, applicants for a pre-symptomatic test first see a clinical geneticist and a psychologist. The clinical geneticist discusses the history of the disease and the personal reasons for a pre-symptomatic test. Then there is a meeting with one of the psychologists who discusses the implications of pre-symptomatic testing. This is followed by a four-week period to think the matter over, after which the applicant returns for a final discussion and decision about the test.

Although the subject positions created for FAP patients and individuals at risk are clearly different in the two regimes we have described, we may conclude that in both regimes these positions can be understood as the emergent result of standards, routines and protocols shaping, and being shaped by, the everyday practices of medical decision making.

\section{Standardpatients}

In her study of decision making practices, Schermer also observes that when the preferences of patients are taken into account, medical professionals often refer to what they presume patients in general deem as important, that is, to the image of the average or "standard" patient. This observation likewise applies to the efforts of those involved in the FAP-network. As medical specialists have been increasingly convinced of the necessity to regularly screen members of families at risk of contracting FAP, they have created new courses of action for patients and their relatives, and thus also new opportunities of choice. However, in adhering to the rules and standards of a regime of prevention, medical specialists are not seeking more room for choice, but are seeking opportunities to improve care. The course of action indicated is de- 
fined by the image of a standard patient who wants to know about his or her risks. Indeed, diagnosis and treatment of and screening for FAP are seen as matters of life (longer) or death (early), and decisions about preventive measures are primarily perceived as medical issues from this perspective (Menko et al. $\left.1999^{\mathrm{a}}, 1999^{\mathrm{b}}\right)$. Such decisions thus will be generally discussed and presented in terms of necessary interventions about which there is little scope for choice. Reflecting on his discussions with FAP patients in the consultation room, a gastroenterologist comments:

\begin{abstract}
The days that the doctor knew best are long gone. Choices and risks related to the timing and nature of surgical interventions all have to be clearly discussed with the patient. There are a lot of things that have to be considered, including of course the wishes of the patient.... Of course, when a colon is full of polyps, it is our task to deliver the message, to say what must be done, and that is, you know, what always will be done.
\end{abstract}

Of course, not everybody conforms to the image of the standard patient. Sometimes a patient is not prepared to inform other members of the family. And not everyone at risk really wants to be informed. In living their lives and their disease, people thus may choose to follow different trajectories. However, with the emergence of a practice of clinical screening and its development into a cancer genetic regime, some trajectories have been made more comfortable and predictable than others. In the early 1980 s, it required a great deal of effort for a medical specialist to maintain a program of screening that would allow every individual at risk to be informed and undergo regular examination. Today, a national registry, social workers who visit patients at home, information leaflets, a system of reminders, working groups, survival rates, guidelines form the constitutive elements of a regime of prevention, offering patients and relatives at risk a course of action that is difficult for them to refuse.

In the regime of self-determination, we indeed find another image of the standard patient as someone who has a great stake in self-determination and non-interference. Thus, in the day-to-day practice of clinical genetics, everything is done to uphold non-directiveness and informed freedom of choice. But, as we have noted above, the presumptions made about the values and preferences of the standard patient need not always match the wishes or views of the individuals involved. For example, in moving through the FAP-network, many patients or individuals at risk experience the genetic centre as "just a stop on their way to the surgeon" (Dudok de Wit 1997, 179). They experience their choice as a purely medical decision and sometimes feel annoyed 
by appointments with a clinical geneticist or psychologist taking time to explain the implications of predictive testing. ${ }^{9}$ As Schermer already noted, autonomy may have many different faces indeed!

\section{Conclusion: Where do the choices lie?}

In our description of the emergent FAP-network in terms of two co-existing regimes, we have focussed on a particular contrast in the ways in which roles and responsibilities are assigned to patients or individuals at risk. In a regime of prevention, individuals are presumed to act as obedient clients with an interest in strategies to prevent future disease, whereas in a regime of self-determination, their role is one of autonomous decision makers, capable of articulating their own will and making use of their right to freedom of choice. In discussing these different subject positions we did not start from a particular notion of the human subject. We have described these positions as constituted in particular practices, that is, as being shaped by a variety of standards, routines, guidelines, forms and services. Thus, in our account, individual autonomy is not taken as a normative and political ideal to be contrasted with the realities of everyday practice. We see the act of self-determination as one of the possible, collective outcomes or effects of the association of a sociotechnical network. In the words of Annemarie Mol, we are talking about ontological politics, in which "the crucial moments are not those where 'patients' act as agents, but rather those where they are defined, measured, observed, listened to, or otherwise enacted" (Mol 1999, 87).

Our perspective marks a significant distance from a normative position that starts from a conception of the human subject as a rational, liable and accountable being, thus presupposing freedom of choice is a fundamental condition of existence. From this position, autonomous decision making serves as a universal normative ideal, and the question as to whether individuals have been able to realise their autonomy is considered the major issue in any normative discussion. In our analysis, however, we have tried to move away from dualistic accounts in which autonomy is opposed to paternalism, nondirectivity opposed to directivity, and free choice opposed to forms of pressure. We are interested in the questions of where, when and how choices and autonomy appear as part of a story in which the "good" may have many different faces and need not be necessarily related to autonomous decision making (Mol 1997). Thus, in the consultation room of the gastro-enterologist, choices may appear as a valuable option when discussing the operation date with a young FAP patient who first wants to finish school and find a job. But in 
another situation - when a colon is full of polyps - the doctor, in the words of a gastro-enterologist, simply will have to specify what must be done. In the genetic centre, choices indeed are the trademark of a clinical geneticist. Nevertheless, when the consequences are very straightforward, as with FA P, and somebody does not want to talk and just goes for a blood test, one need not, in the words of a counsellor, always adopt a "holier-than-thou" attitude (interview data, see footnote 8 ).

Seen from this perspective, the question is not how to evaluate the different practices and regimes in an FAP-network in terms of freedom of choice as a universal normative point of view. Rather, we suggest that in order to understand and evaluate the normative implications of medical practices, we need first of all to understand what "choice" requires and implies in various contexts and thus to study the various ways in which choice may, or may not, become an issue in a variety of local and specific situations.

\section{Notes}

1 We would like to thank Brenda Diergaarde and Annemarie Mol for their contribution to the research that was performed in order to write this article.

2 In practice, however, the option of prenatal diagnosis appears to be rarely used (Whitelaw et al.1996).

3 According to Rip and Kemp $(1998,338)$ a technological regime may be defined as "the rule-set or grammar embedded in a complex of engineering practices, production process technologies, product characteristics, skills and procedures, ways of handling relevant artifacts and persons, ways of defining problems - all of them embedded in institutions and infrastructure".

4 This does not imply that other medical practices are naturally directive. What we argue here is the particular emphasis on patient autonomy in the definition and practice of clinical genetics. Whether a non-directive approach is actually possible is another matter. For a critical review of non-directiveness and neutrality, see Van Zuuren (1996, 1997), Steendam (1996), Michie et al. (1997).

5 Paternalism we take here as an act that is performed independent of the wishes of another but with the intention to act to the benefit of that other (Ten Have et al. 1998).

6 See, for example, Berg and Mol (2001), Mesman (2002), Schermer (2001), The (1997).

7 For example, Schermer (2001) argues that as patients often leave the decision making in the hands of what they consider competent professionals, these professionals will experience a moral duty not to harm the trust placed in them. 
8 The quotations in this section are taken from interviews with a social worker from the Foundation for the Detection of Hereditary Tumours, a clinical geneticist from the Centre of Clinical Genetics in Leiden, and a gastroenterologist from the University Hospital in Nijmegen.

9 Also, the opposite experience of patients has been noted. For example, in his study of a practice of genetic counselling, Charles Bosk observes that when parents had to make a choice, they often felt left alone by the genetic counselor who in their eyes refrained from taking the responsibility of making the decision with them (Bosk 1992). 